\title{
Super-Resolution Imaging For Objects Within A Half Space Based On Fully Polarimetric Radar
}

\author{
Yan Zuo,Peng Gao \\ Research Institute of Electronic Science and Technology,University of Electronic Science and Technology of China \\ Chengdu,611731,China,penggao@uestc.edu.cn
}

Keywords: radar imaging; fully polarimetric radar; half space

\begin{abstract}
This paper is based on the application requirements of fully polarimetric radar for near ground and sea targets detection, super-resolution radar imaging has been conducted in this paper, based on an efficient method for the analysis of electromagnetic scattering from 3-D objects within a half-space. By truncating the interface, the analysis of scattering from an object within a half-space can be cast into that of a composite (high contrast) dielectric object in the free space, resulting in an efficient and accurate method by avoiding the numerical evaluations of Sommerfeld integrals totally. Using the calculated fully polarimetric far field scattering data, radar imaging of simple manmade targets has been conducted by a super-resolution imaging algorithm. Differences of electromagnetic scattering characteristics of some manmade targets with different heights above ground or sea and the radar images are also have been compared. Numerical results are presented to demonstrate the effectiveness and accuracy of the proposed method for the analysis of electromagnetic scattering and the identification of targets near the half-space interface.
\end{abstract}

\section{Introduction}

Fully polarimetric radar is a hot research topic in the academic and engineering fields [1] [2]. There is target related information in the scattering field which can be used to identify the geometry, position and even the medium parameters of the target. Polarization is one of the essential characteristic of electromagnetic (EM) wave. In [3] and [4], the polarimetric information of electromagnetic wave can be added to expand the dimension of signal which can be used in radar target recognition. At present, most of the scholars have not taken into account the use of polarimetric information and the effects of ground and sea. The research work in this paper is based on the practical engineering application and some theoretical study of fully polarimetric radar and half space scattering problems in [5]. On the other hand, numerical analysis of electromagnetic scattering from 3-D objects within the half-space background is of great interest due to the wide applications, such as the geophysical exploration, the target identification, the remote sensing and so on [6]. Unfortunately, traditional numerical methods for objects within a half space background are always lack of efficiency [7].
Facing the requirements mentioned above, a novel and efficient method has been presented to calculate the fully polarimetric far field scattering data [6]. A super-resolution algorithm based on the orthogonality of the signal subspace and the noise subspace, the multiple signal classification method (MUSIC) [8], has been used to extract scattering information of the targets in a half space background. Numerical results demonstrate the effectiveness of the proposed method and the influence of the background on the radar imaging or targets detection.

\section{Numerical model for targets within a half space}

Consider the scattering of an arbitrary object in a half space. The half-space, separated by a planar interface, consists of a lossless upper region, usually air or free space, and a dielectric lower region with its high-contrast parameters. Considering the open interface closed at the infinity or the points sufficient far away from the object, we replace the semi-infinite lower region with a closed dielectric region. In other words, the infinite interface has been truncated to the finite one with sufficient large radius, and the interaction between the object and the part of the interface outside the truncation is negligible. Therefore, the original half-space problem is simplified as an equivalent problem of a composite dielectric object scattering in the free space [6].

Consider time-harmonic ( $\left.e^{-i \omega t}\right)$ EM scattering by a composite object, donated by $D_{l}$ with constant electromagnetic parameters $\varepsilon_{l}$ and $\mu_{l}$, which $l=1$ and $l=2$ represent the target and region 2 of the composite object respectively. The surface between the domain $D_{l}$ and free space is denoted by $S_{l+}$ while $S_{l-}$ denotes the internal surface of the domain $D_{l}$, and $n_{l}$ denotes the outward unit normal vector of $S_{l+}$. Moreover, the interface between the domains $D_{1}$ and $D_{2}$ is defined as $S_{3}$, and $n_{3}$ is the unit normal vector pointed from $D_{2}$ to $D_{1}$. More details about the definitions have been shown in Fig.1. For the sake of the general, the target is considered as a dielectric object, and the solving equations can be formulated by extinction theorem, written as followed as eq. (1). 


$$
\left[\begin{array}{cccccc}
\eta_{0} L+\eta_{1} L_{1} & -K_{0}-K_{1} & \eta_{0} L_{0} & -K_{0} & -\eta_{1} L_{1} & K_{1} \\
K_{0}+K_{1} & \frac{1}{\eta_{0}} L_{0}+\frac{1}{\eta_{1}} L_{1} & K_{0} & \frac{1}{\eta_{0}} L_{0} & -K_{1} & -\frac{1}{\eta_{1}} L_{1} \\
\eta_{0} L_{0} & -K_{0} & \eta_{0} L_{0}+\eta_{2} L_{2} & -K_{0}-K_{2} & \eta_{2} L_{2} & -K_{2} \\
K_{0} & \frac{1}{\eta_{0}} L_{0} & K_{0}+K_{2} & \frac{1}{\eta_{0}} L_{0}+\frac{1}{\eta_{2}} L_{2} & K_{2} & \frac{1}{\eta_{2}} L_{2} \\
-\eta_{1} L_{1} & K_{1} & \eta_{2} L_{2} & -K_{2} & \eta_{1} L_{1}+\eta_{2} L_{2} & -K_{1}-K_{2} \\
-K_{1} & -\frac{1}{\eta_{1}} L_{1} & K_{2} & \frac{1}{\eta_{2}} L_{2} & K_{1}+K_{2} & \frac{1}{\eta_{1}} L_{1}+\frac{1}{\eta_{2}} L_{2}
\end{array}\right]\left[\begin{array}{l}
J_{1} \\
M_{1} \\
J_{2} \\
M_{2} \\
J_{3} \\
M_{3}
\end{array}\right]=\left[\begin{array}{l}
-\vec{E}^{i} \\
-\vec{H}^{i} \\
-\vec{E}^{i} \\
-\vec{H}^{i} \\
0 \\
0
\end{array}\right]
$$

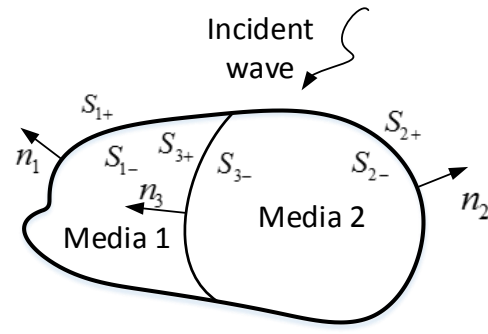

Fig.1 Detailed description for the new model

\section{Radar imaging theory}

\subsection{Range-doppler algorithm for inverse synthetic} aperture radar imaging

The backscattered electric field from a target can be approximated as the sum of scattering from a finite number of single scatters, called scattering centers. Then, the small-bandwidth and small-angle ISAR image of the target can be found by taking the $2 \mathrm{D}$ inverse Fourier integral of the 2D backscattered field data as

$$
\begin{aligned}
\operatorname{ISAR}(x, y) & =\boldsymbol{A}_{0} \cdot \mathcal{F}^{-1}\left\{e^{-j 2 \pi\left(\frac{2 f}{c}\right) x_{0}}\right\} \cdot \mathcal{F}^{-1}\left\{e^{-j 2 \pi\left(\frac{k_{c} \Phi}{\pi}\right) y_{0}}\right\} \\
& =\boldsymbol{A}_{0} \cdot \delta\left(x-x_{0}, y-y_{0}\right)
\end{aligned}
$$

where $\left(x_{0}, y_{0}\right)$ is the position of a scattering center and $A_{0}$ stands for EM reflectivity coefficient. Since the 2D ISAR image is nothing but the display of range profile in one axis and cross-range profile in the other axis, it is intuitionistic and famous as RD (Range-Doppler) algorithm [9].

\subsection{Two-dimensional super-resolution imaging algorithm}

The super-resolution imaging algorithm based on MUSIC method needs to extract the information of scattering points of a target, so it is necessary to establish a parametric scattering model. The GTD (Geometrical Theory Diffraction) model is more suitable to get more accurate information in this case. According to Ref [10], the two-dimensional GTD model is:

$$
x_{p q}(f, \theta)=\sum_{i=1}^{d} s_{i, p q} \exp \left(-j 2 k\left(x_{i} \cos \theta+y_{i} \sin \theta\right)\right)+u_{p q}(f, \theta)
$$

where $p q$ stands for different polarimetric channels, which are $\phi \phi, \phi \theta, \theta \theta$ and $\theta \phi$ in spherical coordinate system. $\theta$ is aperture angle. $x_{i}$ and $y_{i}$ is the position of a scattering center. The projections of wave number on $\mathrm{x}$-axis and $\mathrm{y}$-axis are $k^{x}$ and $k^{y}$.

The MUSIC spertrum is

$$
P_{2 D}(x, y)=\frac{a(x, y)^{H} a(x, y)}{a(x, y)^{H} U_{N} U_{N}^{H} a(x, y)}
$$

where $U_{N}$ is noise subspace and the steering vector is

$$
a\left(x_{i}, y_{i}\right)=\left[\begin{array}{c}
\exp \left[-j 2\left(k_{1}^{x} x_{i}+k_{1}^{y} y_{i}\right)\right] \\
\exp \left[-j 2\left(k_{2}^{x} x_{i}+k_{1}^{y} y_{i}\right)\right] \\
\ldots \\
\exp \left[-j 2\left(k_{M}^{x} x_{i}+k_{N}^{y} y_{i}\right)\right]
\end{array}\right]
$$

\section{Numerical results}

\subsection{One-dimensional high resolution imaging}

The High Resolution Range Profile (HRRP) of radar targets can be obtained easily, and it is widely used in engineering application. A CAD model has been created, as shown in Fig. 2

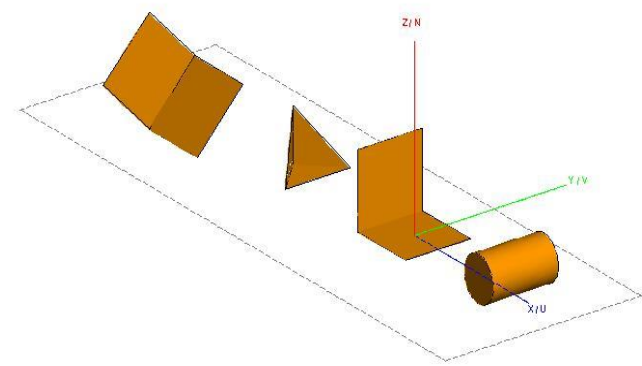

Fig.2 CAD model of simple targets

The central positions of these targets are on $\mathrm{x}$-axis. They are $(3,0),(-1,0),(-5,0)$ and $(-9,0)$. The incident angle of fully electromagnetic wave is $\mathrm{phi}=0^{\circ}$, theta $=45^{\circ}$. The simulated monostatic scattering data were generated from $\mathrm{f}=1 \mathrm{GHz}$ to $\mathrm{f}=1.3 \mathrm{GHz}$ in equally space frequency points without any noise. The radar images of these targets with different heights of $5 \mathrm{~m}$ and $10 \mathrm{~m}$ are shown in Fig.3 and Fig.4, respectively.

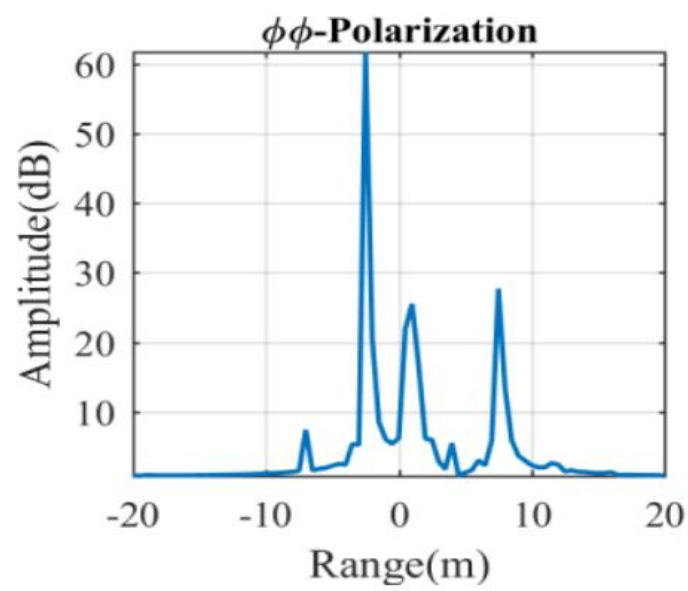

Fig. 3(a) Radar images of targets with the height of $5 \mathrm{~m}$ above the sea surface (HRRP) 


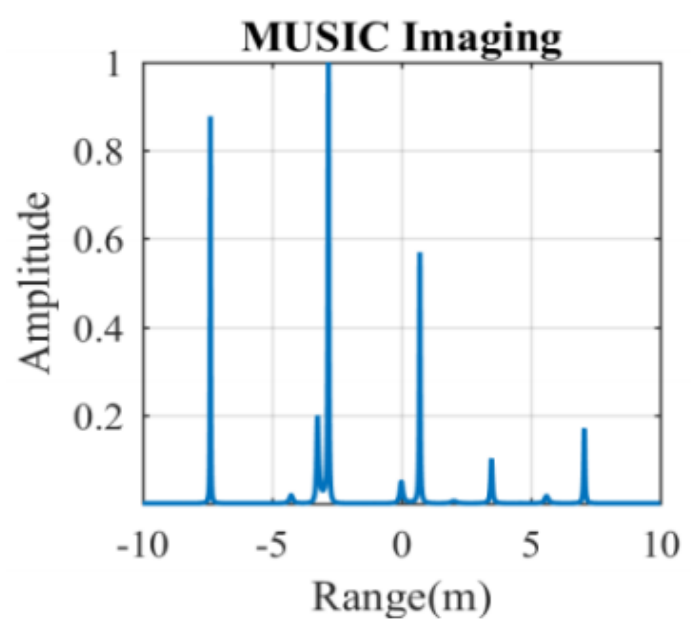

Fig. 3(b) Radar images of targets with the height of $5 \mathrm{~m}$ above the sea surface (MUSIC Algorithm)

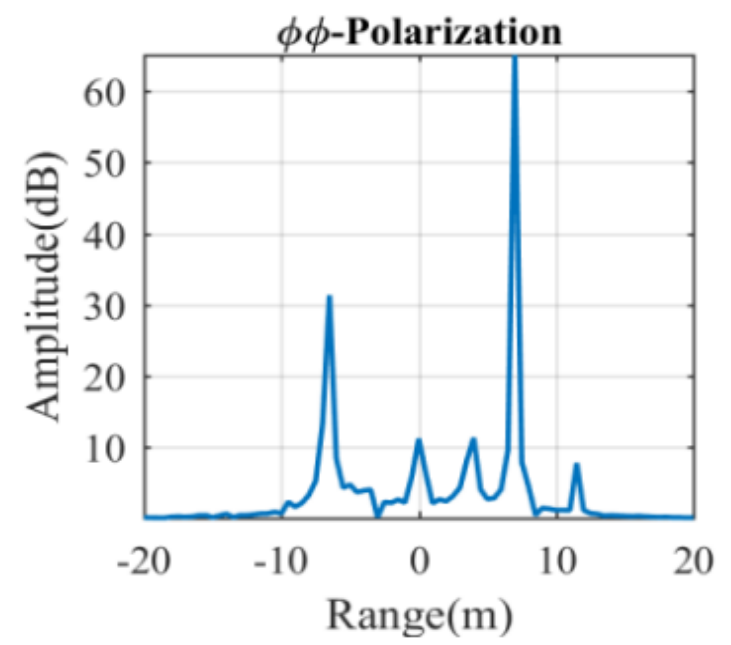

Fig. 4(a) Radar images of targets with the height of $10 \mathrm{~m}$ above the sea surface (HRRP)

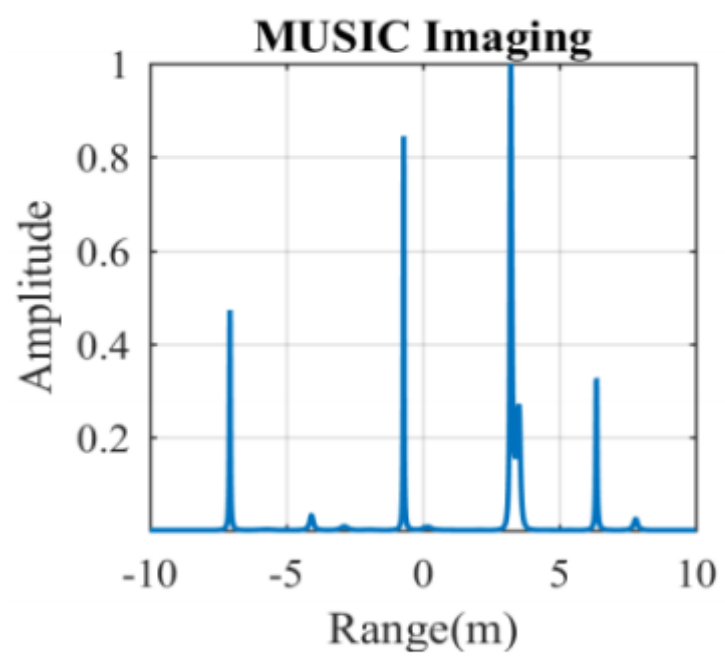

Fig. 4(b) Radar images of targets with the height of $10 \mathrm{~m}$ above the sea surface (MUSIC) Algorithm

In Fig. 3(a), there are 3 higher peaks among all the 5 peaks which represent a cylinder, a dihedral reflector and a trihedral reflector. In Fig. 4(a), we can find there are 2 peaks with higher amplitudes. But there are still 3 another peaks. We cannot identify which are true targets and which are the peaks caused by the coupling effects. In Fig. 3(b), the positions of reflectors can be seen clearly and accurately. Even if these targets are above sea or ground, we can still recognize the positions of scattering points, as shown in Fig. 3(b) and Fig. 4(b). The MUSIC method is more effective in extraction of scattering centers. But the positions may be shifted by the coupling effects between targets and environment. This is more obvious in two-dimensional ISAR imaging.

\subsection{Two-dimensional imaging for targets within a half-space}

In this section, some numerical examples are given to show the influence of the background on the target detection and radar imaging. A sphere array has been established with the central positions are $(1,0,1),(0,1,1),(0,-1,1),(-1,0,1)$ and $(0,0,1)$, shown as fig.5. The bandwidth of the fully polaricmetric incident wave is $500 \mathrm{MHz}(7.25-7.55 \mathrm{GHz})$, and the incident angle is $\theta=45^{\circ}, \phi \in\left[-2^{\circ}, 2^{\circ}\right]$. The numbers of frequency sampling points and angle sampling points are 33 and 17 respectively. The imaging results of this array within a free space or a half space have been simulated and shown as Figs.6-9.

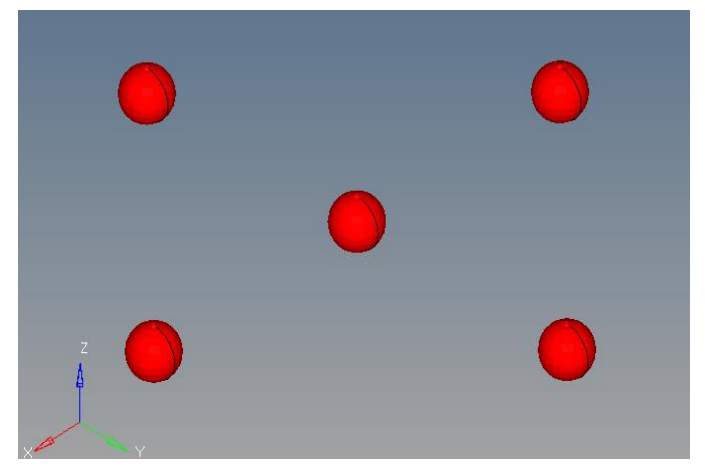

Fig.5 A PEC sphere Array

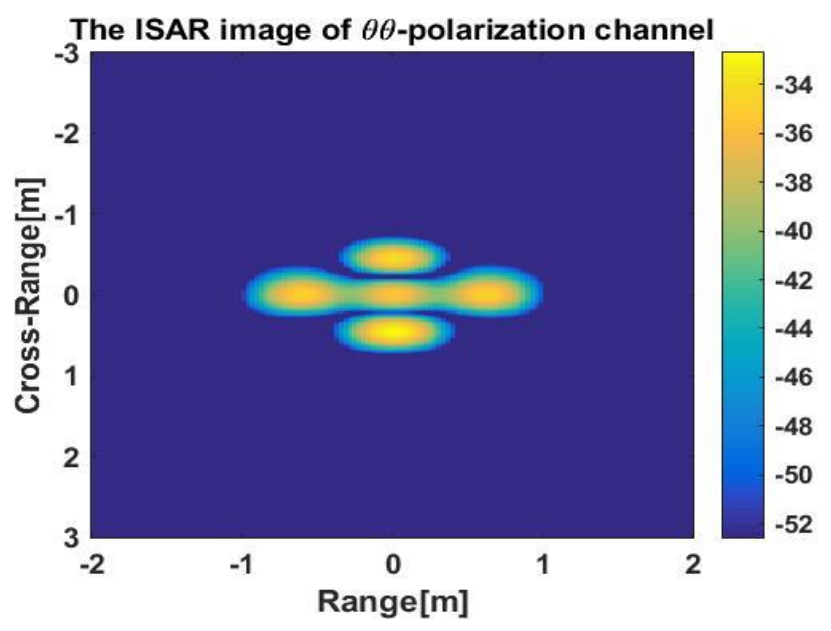

Fig.6 ISAR image of the PEC array within a free space (RD algorithm) 
The super-resolution image of P-MUSIC method

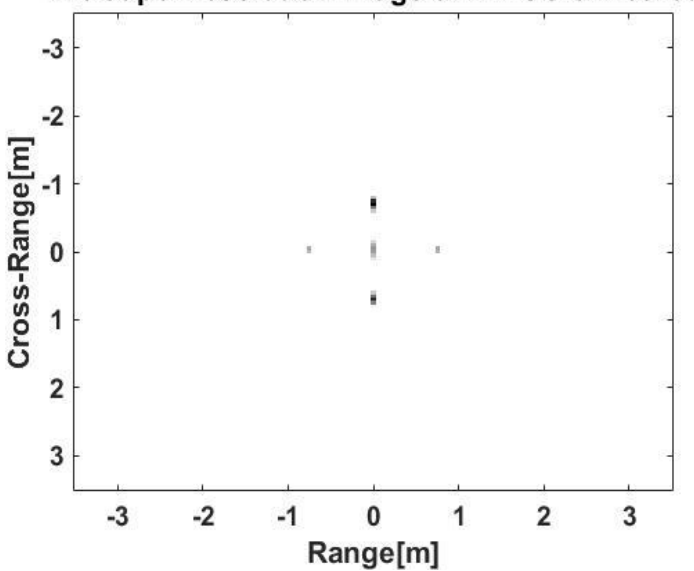

Fig.7 Image of the PEC array within a free space (MUSIC algorithm)

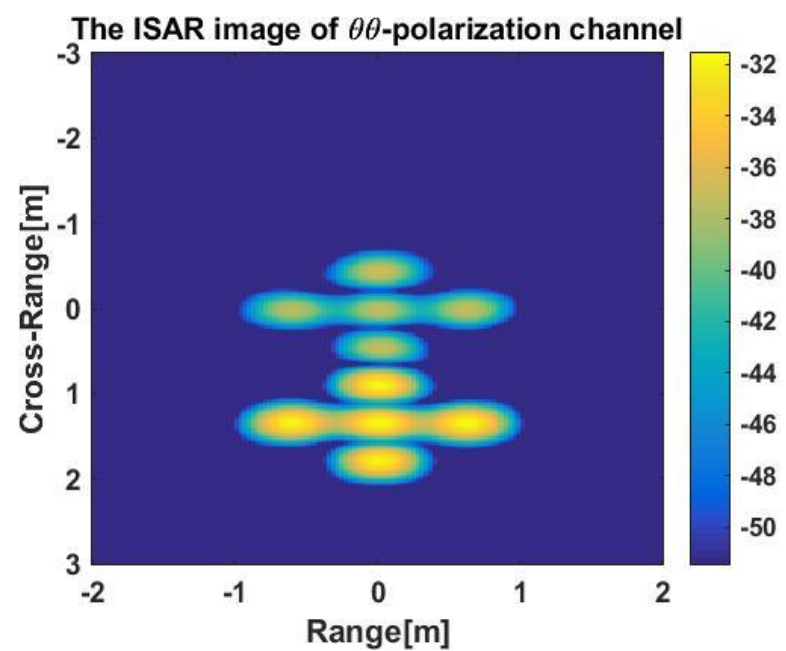

Fig.8 ISAR image of the PEC array within a half space (RD algorithm)

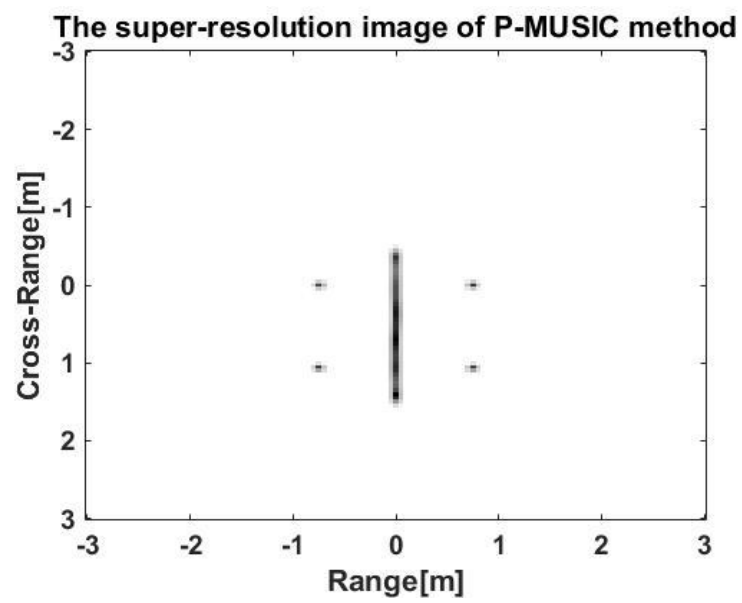

Fig.9 Image of the PEC array within a half space (MUSIC algorithm)

\section{Conclusion}

In this paper, an efficient method to simulate a set of polarimetric radar system has been presented to study the scattering characteristics of simple manmade targets in a half space. The numerical method can be used to calculate the fully polarimetric scattering field. The ISAR imaging has been proposed to detect objects within a half space by using RD algorithm. Moreover, the two-dimensional super-resolution method can be used to extract the positions of scattering points and weaken the coupling effects between the targets and the half space background. Numerical results provide a theoretical reference for radar recognition of targets within a half space in practical engineering.

\section{References}

[1] J. S. Lee, E. Pottier, Polarimetric Radar Imaging: From Basics to Applications, UK: Abingdon, 2012.

[2] Yang. J, "On theoretical problems in radar polarimetry, " Ph. D. thesis, Niigata University, Niigata, Japan, 1999.

[3] Huynen. J. R, "Phenomenological theory of radar targets, " Ph. D. thesis, University of Technologh Delft, Delft, Holland, 1970.

[4] Wang. X. S, "Study on wideband polarization information processing, " $\mathrm{Ph}$. D. thesis, National University of Defense Technologh, Changsha, China.

[5] Xing. S. Q, "Study on 3D imaging of manmade targets based on polarimetric radar, " $\mathrm{Ph}$. D. thesis, National University of Defense Technology, Changsha, China.

[6] X. Qi, Z. Nie, X. Que, Y. Wang, and Y. Yang, "An Efficient Method for Analysis of EM Scattering from Objects Straddling the Interface of a Half-Space," IEEE Geosci. Remote Sens. Lett., vol. PP, no. 99, pp. 1-5, 2016.

[7] Y. P. Chen, W. C. Chew, and L. Jiang, "A new Green's function formulation for modeling homogeneous objects in layered medium," IEEE Trans. Antennas Propag., vol. 60, no. 10, pp. 4766-4776, Oct. 2012.

[8] J. W. Odendaal, E. Barnard, C. W. I. Pistorius, "Two-dimensional superresolution radar imaging using the MUSIC algorithm, " IEEE Trans. Antennas Propag, vol. 42, pp. 1386-1391, Oct. 1994.

[9] Caner Ozdemir, Inverse Synthetic Aperture Radar Imaging with Matlab Algorithms, John Wiley \& Sons Inc, US: New York, 2011, pp. 136-139.

[10] Dai. D, "Study on polarimetric radar imaging and target feature

extraction, " Ph. D. thesis, National University of Defense Technology,

Changsha, China, 2008, pp. 50-56. 